\title{
La poesía quechua: rapto de la escritura y corpus contemporáneo (siglos XX y XXI)
}

Recibido: 10.11 .2018

Aprobado: 25.03.2019
Gonzalo Espino Relucé Universidad Nacional Mayor de San Marcos gespino@unmsm.edu.pe

\section{RESUMEN}

La poesía quechua contemporánea la entendemos como la afirmación de una cultura de resistencia, su vigencia como lengua y como ejercicio de escritura se produce en todo el siglo xx y se afianza en el siglo xxi. Se trata de un tipo de texto que se realiza en el entramado de la tradición quechua y la impronta de sus aprendizajes de la cultura occidental y al mismo tiempo pone en primer plano cómo y con qué criterios se abordaría el corpus de la poesía quechua escrita contemporánea. Su abordaje tendría que ser revisada desde el rapto de la escritura que implica atender a nociones como proceso, escritura y zona literaria. Se trabajó sobre un universo de fuentes que va de 1904 al 2017.

Palabras Clave: poesía; quechua; rapto de la escritura; proceso; zona literaria; Perú

\section{Quechua poetry: kidnapped writing and contemporary corpus (XX and XXI centuries)}

\begin{abstract}
We understand contemporary Quechua poetry as the establishment of resistance culture. Its validity as written language occurs through the twentieth century and strengthens in the twenty-first century. As a text type made in an entanglement of Quechua tradition with learning impressions of Western culture, we examine how and which criteria could be used to address a corpus of contemporary Quechua written poetry. We reviewed this approach from a perspective of kidnapped writing, considering notions as literary process, literary writing, and literary zone. We worked with sources from 1904 to 2017.
\end{abstract}

KEYwORDs: poetry; Quechua; kidnapped writing; literary process; literary zone; Peru

$1 \quad$ Este artículo forma parte de los resultados del proyecto de investigación Proceso de la poesía quechua contemporánea (2017) y Proceso de la poesía quechua contemporánea II Educación y poesía (2018), proyectos financiados por Vicerrectorado de Investigación y Posgrado UNMSM, del grupo de investigación Discursos, representaciones y estudios interculturales (EILA)-UNMSM, que coordino. 
$\mathbf{P}$ artamos de una confirmación: el quechua es una lengua viva en todos en los Andes de América del Sur, es preferentemente hablada y su tradición oral continúa vigente. Las manifestaciones poéticas son prácticas comunes a todos los pueblos, el caso quechua no es la excepción. Su larga trayectoria se expresa en la vigencia de las diversas formas de su tradición oral. La gran tradición poética quechua pertenece a las aras populares y a las comunidades donde se vuelve a decir en todas sus formas sopesando la memoria y continuidad; renovadas, en nuevos escenarios sociales y naturales, con nuevas sensibilidades. En esta ocasión no hablaremos de la tradición oral quechua, ni de su poesía oral, es decir, aquellas muestras poéticas que se identifican con las formas de canción, o más precisamente con el poema-canción, que se asocia a la música y la danza. Exploraremos un fenómeno nuevo que llama la atención por su renovada presencia en tanto textualidad que se vincula, pero resulta autónoma de la tradición, que testimonia las diversas sensibilidades del sujeto y la escena social, me refiero a la poesía quechua escrita contemporánea, de estos tiempos. Nos proponemos ofrecer una lectura del proceso de la poesía quechua contemporánea escrita. Esto es aquella que ha llegado al dominio de la letra que cuestiona el tópico de lo ágrafo en el caso de las lenguas indígenas.

\section{¿Lengua ágrafa? La letra colonial}

La literatura quechua tiene larga data, se pierda en su propia historia y en términos contemporáneos su presencia como voz y letra. Se corresponde con el devenir de la historia andina, la de un pueblo que terminó organizando una de las civilizaciones más importante del globo. La Inca. Literatura, sí, en el sentido de aquella palabra que no solo denota, sino que construye universos inevitablemente originales, mejor aún una palabra identificada como el "goce de la palabra", una expresión del narrador Carlos Eduardo Zavaleta. Si actualmente resulta imposible descifrar la "letra» del kipu, soportes semióticos de la palabra quechua, estas fueron consignada desde la presencia hispana en la letra que significó desde 1532 violencia, muerte y despojo para la población indígena del territorio andino. Por lo que propongo que convengamos que su existencia no presupone la exclusividad de la escritura sino su continua omnipresencia en el universo oral en la vida de la gente, comunidades y pueblos andinos como formas discursivas de diversa naturaleza: se narra, se canta, se rinde culto a los dioses tutelares, se goza de alegría, se llena de llanto, etc. Sus diversas manifestaciones aparecen en la vida cotidiana y ritual de la gente o han sido consignados de alguna manera en los inventarios, crónicas y relaciones indígenas ante tribunales civiles y eclesiástico. Los soportes siguen siendo un problema, que grosso modo, se reducen a dos mecanismos: la fijación por la letra y la trasmisión oral como manifestación de resisten, durante la colonia y luego republica y su continúa reinvención y renovación en los contextos actuales.

El tiempo, en nuestra lectura, se asocia a lo que la narrativa oral encontramos. Un tiempo en que no existían los Españas y un tiempo en que aparecen los Espańas con una nueva manera de organizar el mundo, las relaciones, con sus nuevas costumbres. Cuando se habla, en la ciudad, de literatura se insiste en la preexistencia de la escritura, es decir, si esta está notificada por algún documento escrito y no necesariamente por el archivo de la memoria que puede ser un quipu, un tejido, un ceramio, un diseño, etc. Siendo así, consideremos la escritura alfabética la convirtió en la lengua de la colonia. El quechua fue identificada como la lengua general, fue registrada y estudiada, se la divulgó y fue utilizada para el trato colonial con las elites indígenas y para la divulgación de la doctrina cristiana, esta tendría un temprano desarrollo con la «Plática para todos los indios // «Llapa runaconapac conasca» (Santo Tomás 1560: [87]96), pasando por los testimonios del Inca Garcilaso, Guaman Poma, el Manuscrito de Huarochirí y todo el teatro quechua colonial (Ollantay, Usar Paukar) a los cancioneros recogidos a finales del siglo XIX.

Respecto a lo primero, podemos establecer que, en líneas generales, esta se encuentra localizada en las primeras crónicas que dan cuenta de la existencia de diversas expresiones que se asocian a la literatura. Es decir, al consignarse el tema de la lengua, el inventario comprometió también indicar si el idioma era apto o no para las expresiones del espíritu y eventualmente, se registra. De este modo se identifica al quechua como la lengua general que tiene "abundancia de vocablos", una lengua, al decir de fray Domingo de Santo Tomás, "conueniencia q tienen con las cosas q significan. Las 
maneras diuersas y curiosas de hablar. El suaue y buen sonido al oydo de la pronunciacion della», para luego calificarla como «Lengua pues, S. M, tan polida y abundante» (...) polida y delicada» (Santo Thomas, 1560), el quechua se puede llamar galana y definir como apta para expresar sentimientos y abstracciones.

Los testimonios tempranos vienen del Inca Garcilaso de la Vega y el Felipe Guaman Poma de Ayala. Para el Inca Garcilaso tenían un gusto clásico, de inspiración renacentista; en el sentido también de validar la cultura inca en el espacio europeo; dirá que «De la poesía alcaçaron otra poca, porque supieron hazer versos cortos y largos, con medida de sílabas», pero lo más característico será su aseveración «Los versos eran pocos, porque la memoria los guardasse, empero muy compendiosos, con cifras; todos eran sueltos.»(Garcilaso Inca [1609]1985:87); precisamente para que la memoria las pueda retener y al mismo tiempo establece una plataforma moderna, el quechua tiene equivalente en la lengua de transacciones de la época, el Latín y la del imperio, el romance. "[p]ara los que no entienden indio ni latín me atreví a traducir los versos al castellano" (:88). Este dato tiene especial relieve, toda vez que se trata de una comprensión que equipara las posibilidades de la poesía quechua como acto universal. Este tono variará en el caso de Guaman Poma que se traduce como práctica común en todos los segmentos sociales y territorios del Tahuantinsuyo; como un ejercicio extensivo, entre "yndios comunes» y cuyas expresiones son «no tiene(n) cosa de hechisería(n) ni ydúlatras ni encantamiento, cino todo huelgo y fiesta, rregocixo.» (f. 315 [317] $)^{2}$ las mismas que va acompañadas de formas de expresiones que se diferencian una de otras formalmente, aunque le incomode y recuse el relajamiento de las costumbres: "Ci no ubiese borrachera, sería cosa linda.». De allí que se trata de dos versiones complementarias que dan cuenta de las comprensiones desde el centro del poder y desde la periferia para el tiempo del gobierno inca y la casi primera centuria de presencia hispánica.

Buen tramo de este periodo lo constituye la escritura colonial destinada a la predica evangélica, al respecto puede revisar toda la producción que se deriva del III Concilio Limense (1582-1583). El quechua se

2 Cf. Felipe Guaman Poma, El primer nueva corónica y buen gobierno (1615-1616) en El sitio de Guaman Poma http://www.kb.dk/permalink/2006/poma/info/es/frontpage.htm. prestigia como parte del renacimiento inca, aparecerá un teatro que testimonio la galana lengua y sus preocupaciones religiosas y sociales, tal como ocurre en el Usca Paucar y Apu Ollanta (Meneses 1983). La continuidad de esta se apreciará años más tarde en el desarrollo del teatro quechua de fines del siglo xix y xx tal como lo han estudiado Itier (1999-2002) para el caso cusqueño y Durston (2004) para el ayacuchano. Hacia la segunda mitad del siglo XIX aparece un notable interés por recopilar las cosas de indios, especialmente sus expresiones musicales que identifico como poema-canción como los de Midderdorf o a inicio del siglo xx los esposos D’Ancourt. Más tarde ya en la República, dos estudios darán cuenta de la existencia de la poesía quechua, la tesis sanmarquina La poesía en el imperio de los incas (1873) de Asciclo Villarán y la Gramática Quechua (1874) de José Dionisio Anchorena, que le dedica toda una sección al análisis de la poesía quechua. En efecto, en el siglo XIX, se encuentran registros poéticos, pero no corresponde al dominio del quechua sino al misti o pertenecen a la órbita del quechua domesticado y colonial, se escribe sobre los indios (Espino 1999 y 2007).

\section{¿Problemas teóricos?}

La poesía quechua tiene como marco de referencia la diversidad lingüística y cultural, 47 pueblos y lenguas reconocidas (Minedu 2015) y las desigualdades que socialmente la acompañan, así como una historia zigzagueante e inesperada. Producida en el marco de un esquema de crecimiento que oscilan entre la pobreza y la estabilidad económica que supone los beneficios del estado y de su modernidad llega a la ciudad, que excluye a los pueblos y comunidades del interior del país.

Entre tanto no debe perderse de vista que una literatura participa del juego tenso y desigual de las hegemonías culturales que en los tiempos actuales, aparece bajo la inofensiva asepsia de la categoría canon instalada por el mercado internacional del texto. Al mismo tiempo reparamos en los ejercicios de poder que se producen en la esfera de las representaciones simbólicas que han terminado por reconocer y validar aquella literatura que se produce en espańol y que margina a las otras literaturas. Estas producciones se contextualizan con las literaturas que se producen en el Perú, hablamos así de diversos sistemas en un espa- 
cio definido como heterogéneo tal como nos lo hacía ver Antonio Cornejo Polar (1983) y como lo ha precisado Carlos García-Bedoya (2014). Lo que se lee, se acepta y difunde desde la esfera oficial y oficiosa: en la escuela, en la Academia y en los medios de comunicación se asocia al libro o formato virtual, preferentemente escrito y en espańol. Esta literatura que, en líneas generales, la identificamos como canónica, dominante y excluyente, que pertenece al mercado del libro y que divulgan las corporaciones de libreros (Ferias, texto escolar), constituye la literatura hegemónica. Las antologías nacionales son una clara muestra de la prevalencia del monolingüismo castellano, no las incorpora y se extendió a las colecciones de poesía indígena realizadas en el siglo xx, si bien cumplieron la función de evidenciar su existencia, difundieron la poesía quechua en español. La Antología general de la poesía peruana (1957) de Alejandro Romualdo y Sebastián Salazar Bondy, incluye una sección de poesía quechua y como poeta contemporáneo a Kilko Warak'a. Las dos de más influyentes antologías poéticas no lo hacen: Poesía Peruana, Antología de la Poesía Peruana (1973) de Alberto Escobar y la Poesía peruana: Antología general. De Vallejo a nuestros días (1984) de Ricardo González Vigil trabajo que considera una «muestra» de la poesía «en lengua española y manifestación escrita». González Vigil lo hará recién en este siglo en Poesía Peruana del siglo XX (Lima: COPE, 1999 , t. I), en ella incluye a los tres poetas fundadores de la tradición quechua contemporánea, me refiero a Kilko Warak'a, Kusi Paukar y José María Arguedas. Las antologías de poesía quechua dudan sobre la pertinencia de la lengua, proponen una imagen histórica y de los poetas contemporáneos que casi siempre se detienen en Kilko Warak'a y José María Arguedas, al respecto pueden revisar Poesía quechua (1965) de José María Arguedas; Poesía y prosa quechuas (1968) y Literatura quechua clásica (1986) de Francisco Carrillo; Poesía quechua de Sebastián Salazar Bondy (1978) y Literatura quechua (1980).

Cuando hablamos de literatura, demandamos la existencia de un corpus. ¿Existe un corpus de la literatura quechua contemporánea?, ¿es factible hablar de un corpus de la poesía quechua escrita? La repuesta fue siempre afirmativa, de esto dan cuenta las tres más importantes publicaciones del siglo pasado: Literatura Quechua (1980), de Edmundo Bendezú, Poesía aborigen y tradicional popular (1984), de
Alejandro Romualdo, y Poesía quechua escrita en el Perú (1993), de Julio Noriega Bernuy. Estas ofrecen un repertorio que evidencia la existencia de una escritura quechua en progresión; difieren en sus referencias espacios-temporales en el balance contemporáneo y la lengua que utilizan. La primera ofrece una selección con apego diacrónico, todos los poemas quechuas provienen del acervo oral, no incluye a poetas de tradición escrita y los textos vienen en español. El mérito de este volumen de la Biblioteca Ayacucho fue que volvió a poner en circulación la poesía quechua en el espacio latinoamericano, junto con la maya, náhuatl y guaraní. La segunda resulta singular al presentarnos un muestrario de las poéticas nativas de los distintos pueblos del país; en él, Romualdo incorpora una sección estructurada de cara a la historia: incluye, para el periodo de la República, diez autores a quienes denomina «Poetas quechuas contemporáneos» (301-384), y los poemas son bilingües (quechua y castellano). Ambos trabajos llegan a 1980. La tercera cubre hasta los inicios de los 80 , abarca ocho décadas del siglo $\mathrm{xx}$ y presenta, por vez primera, autores totalmente desconocidos, y supone la puesta en valor de poetas de circulación local.

No hay duda que la expresión estético literario tiene como material básico el lenguaje y la lengua. La historia es más o menos común, tiene obviamente que ver con el prestigio social de las lenguas, es decir, aquella que se imponen. La escritura terminó por asumir el rol protagónico del ego positivista que adscribía para las literaturas nacionales. La lengua inicial de estas literaturas será el español; de esta manera la producción de una literatura quechua perteneció, groso modo, a la esfera ilustrada del poder y a las elites locales, que dieron lugar a un vasto legado en el teatro colonial, el teatro cuzqueño y ayacuchano de fines del siglo XIX e inicio del siglo xx (Meneses, Itier, Durston), pero no se aventuraron a un ejercicio mayor que disputaran espacios letrados y preferencias poéticas. Más todavía, el estado actual de los estudios de la literatura quechua no ha inventariado ni desarrollado una propuesta que permita una lectura que nos acerque con éxito a la lectura de lo que está ocurriendo con la poesía. Lo que involucra ciertamente una postura teórica y una metodología, que no niegue lo avanzado (Arguedas, Noriega, Huamán Manrique), si no sea capaz de leer el proceso en un escenario nuevo, ya 
no solo del tránsito de la voz a la letra ni del impacto de la modernización, si no la del sujeto y la diversidad de lenguas quechuas, los núcleos de desarrollo Ayacucho, Cusco, Lima y Huaraz, amén de la disminución, en términos absolutos, de la población hablantes quechuas, las redes virtuales y las formas poéticas que consignan estos textos, o las distancias que establecen en un poeta joven y un poeta que empieza a publicar al final de su vida.

\section{Rapto de la escritura}

El rapto de la escritura supuso desplazamientos en la forma como se compone las canciones andinas y la propia poesía que tuvo que reimaginar la situación del indio y el mestizo (Arguedas [1938] 2012), empezando por un asunto básico que se conecta con la modernidad de occidente: el tránsito del ñuqanchik, sujeto colectivo, a un sujeto individual, ñuqa, en el enunciado poético. Entonces, ¿cómo se expresan las miradas colectivas?, ¿cómo estas se manifiestan? La poesía quechua escrita contemporánea tiene su arraigo en el legado quechua que supone memoria y concepción andina del mundo y al mismo tiempo reinvención de formas tradicionales (dístico semántico, humanización de los objetos, etc.); y en el mejor de los casos, la situación de enunciados que representan el sentido colectivo y se manifiestan, a sí misma, de manera autónoma o el posicionamiento de una voz singular e íntima.

Es en la última década del siglo pasado que cobra un impulso inusitado la poesía quechua. La lengua nativa se reivindica, se publican revistas y libros, se desarrollan concursos y se percibe un circuito propio propiciado por comunidades letradas que se extiende a los quechuas residentes en las principales ciudades del país. Los contextos de desarrollo los asocio a un conjunto de hechos: (1) al fin de la guerra interna, que creó un clima favorable para el florecimiento de las letras quechuas que estaban estancadas en las gavetas individuales; (2) la promoción institucional de la literatura quechua desde varias esferas del Gobierno y de las Universidades (concursos Premio Nacional de Literatura Quechua, Cátedra Quechua; publicaciones desde ministerios y gobiernos regionales), que colaborarán en la consolidación de, cuando menos, dos polos de desarrollo para la literatura quechua de estos tiempos (Huamanga y Cusco); y, (3) una época propicia para las lenguas indígenas avalada por los convenios internacionales y la divulgación de las literaturas y culturas indígenas en América Latina. Esto terminaría por dar cuenta de nuevos e inusitados derroteros para la literatura quechua en general. No está de más recordar la extensa tradición oral de las prácticas poéticas andinas que coincide, a fin del siglo $\mathrm{xx}$, con la configuración de la canción andina moderna que viene de los fueros estéticos de Ayacucho (Huamán López 2015).

Planteo tres conceptos operativos para el estudio de la poesía quechua contemporánea: proceso, rapto de la escritura y zona literaria. El proceso se corresponde con la historia y con ello la configuración escritural quechua como de larga duración. Indagamos procesos porque nuestras referencias son diacrónicas y basadas en el largo plazo, entendida como espacio temporal en el que socialmente ocurren hechos de transformación significativos. Como se apreciará no se trata del taki, es decir, la canción ni composición tampoco transcripción de la tradición oral a la letra. Esta pesquisa cubre el periodo que va de 1904 a 2018, es decir, del primer poema escrito en quechua y divulgado en el siglo xx hasta el último publicado en junio del 2017. Con el concepto rapto de la escritura grafico cómo los poetas decidieron escribir en quechua, esa lengua que arrastraba el estigma del indio, el símbolo del atraso y la discriminación social. El rapto será la característica que comportan los poetas y sus poemas a lo largo del siglo Xx y XXI, con especial atención a la primera mitad de siglo xx. El concepto zona literaria lo asocio a lo que Claudia Rodríguez Monarca (2017: 33 ss.) identifica como diversas relaciones que establece una literatura no canónica e instala una movilidad respecto al canon.

El rapto de la escritura a su vez supone el aprendizaje de la cultura de occidente, lo que implica un conocimiento básico de la literatura y cultura de occidente que da la escuela y la universidad. Esta se traducirá en un nuevo ejercicio de escritura. Todos los poetas quechuas estudiados tienen un aprendizaje de la escritura literaria. Será esa extrañeza que convierte al sujeto quechua en el mundo occidental, que para entonces, lo acepta y sabe las reglas de urbanidad, entre ellas la de hablar el idioma de la ciudad y la desborda al empezar a escribir en la lengua del indio. La escuela fiscal cumplirá un rol central. 
El rapto se consuma en la primera mitad del siglo xx, el aprendizaje en la otra lengua, en la escuela que occidentaliza, que mata lo mágico al indígena, que le niega su propia cultura, que le abre al mundo y que le permite acceder a los previsiones sociales y civiles del país. Leer se convierte en una necesidad, escribir una urgencia. Esta escritura ofrece ventajas comparativas con aquello que en los tiempos contemporáneos se guarda en la memoria. Y se corresponde con sectores medios que transitan de los pueblos del interior a la ciudad y, en especial, a la ciudad centro, a la capital del Perú. Es durante los años cincuenta que se produce los cambios. La ciudad se moderniza, atrae, se vuelve mágica para la provincia, allí está lo nuevo, el avance, el progreso. Las elites provincias asumen que la lengua de prestigio es la que se habla en Lima, que el castellano es la lengua del poder, que el quechua pertenece a los indios. Estas elites que hasta entonces hablaban quechua (Baquerizo 1989), la abandona. El quechua por entonces alcanza lo que llamaremos el quiebre histórico. La estadística del censo de 1940 consigna: $51 \%$ de la población habla español; el $47 \%$ lo hace en quechua (INEI Censo 1940). Por entonces, se escribirán lo más significativo de su obra en la lengua que se desprecia, se llama así mismos Kilko Warak'a o Kusi Paukar, son «indios» para comunidad y al mismo tiempo Andrés Alencastre y César Guardia Mayorga, usan su identidad civil, son «mistis» para la ciudad (Noriega 1993).

La idea de zona literaria identifica los procesos que permiten que un texto - poema, poemariocircule de un sistema a otro y viceversa, al tiempo que suele trasgredir el orden establecido. En nuestro caso, nos lleva a cuestionar la periodización que hace coincidir fecha de nacimiento con publicación y que propician un momento de época. El proceso al que aludo no crea ese nexo epocal porque se mueve en otro sistema, en el que se publica, casi siempre, tardíamente y se la entiende como hecho aislado y en el mejor de los casos, una curiosidad de letrados sobre los indios, salvo notables excepciones: J. M. Arguedas comentando la poesía de Kilko W'araka; Manuel Baquerizo la poesía de Dida Aguirre, Isaac Huamán sobre Baltazar Azpur o Julio Noriega sobre los poetas trasandinos). Mejor aún, se sabe que existe, que circula, pero se la oculta (Guillén 2016; Espezúa 2017), se la obvia e invisibiliza.
El caso de Sócrates Zuzunaga Huaita (Páucar del Sarasara, Ayacucho, 1954), uno de los más influyentes narradores andinos, tiene una intensa movilidad que se observar en su perfil autoral. Formado primero en un Instituto Pedagógico Superior, luego en la Universidad Nacional Federico Villarreal se desplaza desde la periferia hacia el centro, sabe de su ubicación marginal y fronteriza, y al mismo tiempo es capaz de transitar por instituciones oficiales y oficiosas, así lo revelan los diversos premios que ha obtenido. Entre estos destacan: Premio Nacional de Educación Horacio, «El Cuento de las Mil Palabras» de la revista Caretas; Premio Nacional de Literatura Quechua, (Cuento 2000) y (Poesía 2006); y, Premio COPE de Oro de la II Bienal de Novela, Premio COPE Internacional 2009. Su narrativa, en la mayoría de los casos, viene en un castellano que se ve impregnada por el quechua. Al mismo tiempo que se moviliza sobre dos géneros, principalmente la narrativa y la poesía. La economía de relato lo definen como un extraordinario narrador, un escritor que domina el ichuk kwintu, relatos breve de un humor pícaro y desbordante, de cuya estirpe es Taita Serapio que tuvo su origen en Anécdotas de Taita Serapio y que fue incluido en libro quechua ganador 2000: Tullpa willaykuna; y su novela La noche y sus aullidos (2010) no solo conmueve por los registros del conflicto armado interno, sino por forma como se construye la propia novela. Lo hace también en poesía, esta vez en quechua: Kuyaypa Kanchariynin y su plaqueta Poesía quechua. Al tiempo que lo hace sobre un referente que ha tomado importancia en las dos últimas décadas, me refiero a la literatura infantil y juvenil: Florecitas de Nawin pukio y otros cuentos, Takacho, Takachito, Takachin, Zorrito de Puna, entre otras. Un detalle adicional es que, como autor, es a su vez el promotor de su propia producción.

\section{¿Corpus de la poesía quechua contemporánea?}

Rolando Álvarez (2017: 15-49) nos ha recordado la compleja y heterogénea realidad de nuestra literatura, más aun lo urgente que resulta imaginar, repensar y organizar un corpus literario, sobre todo si se trata de focalizar nuevos procesos. La conformación de los corpus de nuestras literaturas es una tarea pendiente. Los sistemas de valoraciones y los criterios de selec- 

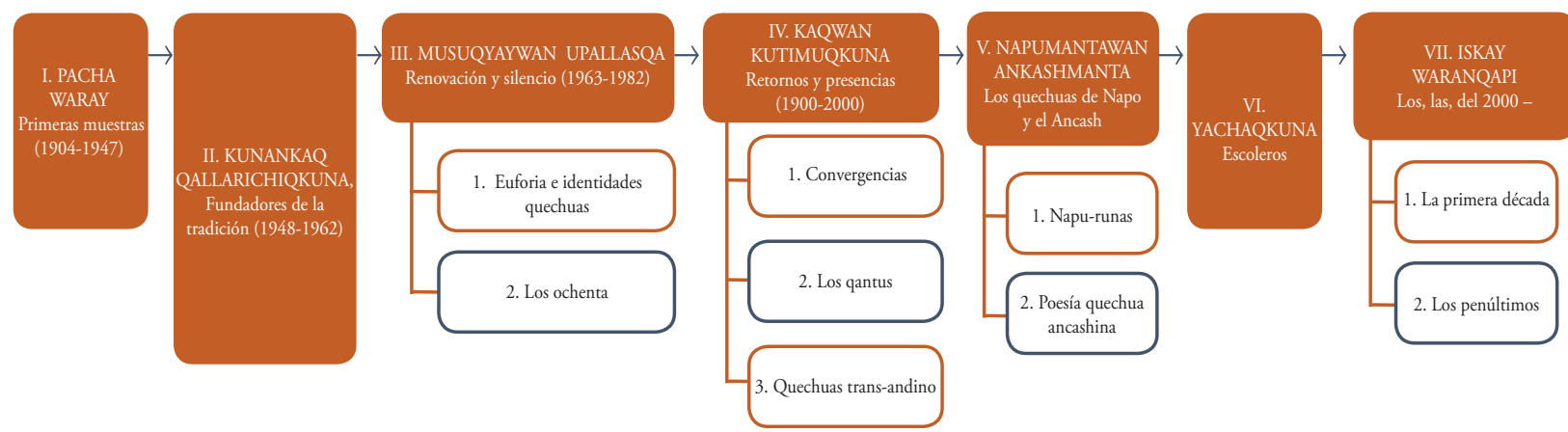

Gráfico 1

ción, en este caso obedece al espesor de la producción poética quechua. De hecho confrontamos varias dificultades que paso a encararlas a partir del desarrollo desigual de la poesía quechua que termina por visibilizar a unos y a otras confinarlas en el olvido. Estas a su vez vinculadas a polos de desarrollo de las aldeas letradas, sobre todo en los años cincuenta del siglo pasado cuando las élites asumen que la lengua de prestigio es el castellano e inversamente empiezan a recurrir al quechua, especialmente en Cusco y Huamanga, como gesto identitario y como reinvención regional. Si esto aparece como lo más expresivo, anotamos que podemos imaginar también los desarrollos tardíos en espacios como Huaraz, Huancayo y Huánuco lugares donde se habla quechua ancashino, huaylash o huanuqueño. En las que, en efecto, encontramos escasas producciones, destacan de manera especial el núcleo ancashino que en los noventa aparecen con algunos textos hasta tener actualmente una representación que bien merece nuestra atención. Distintos serán los casos donde el quechua quedó como expresión básicamente oral, aun cuando las escrituras experimentales estén circulando, estos son los casos de Cañari-Cajamarca, donde casi todo pertenece a la tradición oral. Y lo es todavía más difuso, si se trabaja con el quechua amazónico ${ }^{3}$ donde lo que encontramos son muestras que pertenecen a las tradicionales culturales del acervo oral y que han sido compiladas y que aquí de manera excepcional. En general, la condición de hablantes quechuas permite la escritura en la materna; sin embargo, cuando

3 El quechua amazónico comprende a los «Quichua, Lamas y Kichwaruna las que agrupan un total de 36 mil 163 habitantes", Cf. Familia Lingüística quechua, Censo $2007<$ http://proyectos. inei.gob.pe/web/biblioineipub/bancopub/Est/Lib0944/cap04.pdf> (2.2.2018). hacen suyo el nuuqanchik, tienen que poner sus poemas en castellano. El término que mejor se ajusta es trascreación, es decir, la creación de un nuevo poema a partir de un texto otro, de allí que resulta difícil seguir una traducción literal. Amén de las posibilidades de acceder al texto, sea por compra, intercambio o localización en re positorios bibliográficos, dada el carácter artesanal de las publicaciones.

Esta cartografía poética tiene marca heteróclita, se organiza partir de lo que se ha dicho. Intelectuales que circulan pero que publican tardíamente o segmentos que representan diversos momentos. Será el decurso de una historia accidentada, sorpresiva y zigzagueante, no la hemos imaginada como una historia de sucesos uniformes ni únicos, por eso, la diacronía será intercedida por la sincronía. Si hay una memoria histórica: se pasa revista a la fecha de nacimiento y al momento que el poeta sitúa su data literaria quechua. El gesto todavía no cubre los desarrollos desiguales y las diversas posibilidades de la lengua quechua; por eso que nuestra antología abre dos secciones que pone atención al quechua ancashino y al quechua amazónico, más precisamente, a los quechuas del Napo. A ello agregamos una variante temática, emergente, se trata de la poesía quechua que circula en las escuelas.

El primero de enero 1904, en Tarma, aparece el poema "Cantares/Cantainichicguna» que firma D.S.V. en Aurora - Pacha huarai, el poema tiene un tono indigenista, al mismo tiempo está pensando en los lectores no quechuas. No debe perderse de vista que hacia 1934 se convoca al Concurso de Literatura Kechwa por «el IV Centenario del Cusco»", es decir al cuarto centenario de su fundación española; los re-

4 La revista Waman Puma n. 3 y 4 da cuenta del hecho; publica el "Acjamccaray Ccosccoman, poema kechwa premiado en 1934" (Indio Enelda 1942: 18-19). 
sultados de esa ocasión no tienen mayor trascendencia, salvo porque el regionalismo cusqueño se abre a otras variantes, el poema ganador estaba escrito en "Runa-Simi de Huamanga» (Indio Enelda 1942: 19) y en 1951 se realiza en Cochabamba el Primer Concurso Internacional de Literatura Quechua, que ganará Andrés Alencastre por su poema "Intillimani». Sin embargo, el primer repertorio, en efecto, la identificamos con Canas i sus relámpagos (1947) definida como "Antología poética», que publica a Audaz del Castillo, Killku Warakca, Tupak-Amaro y Jacinto Yana-Aucca, ${ }^{5}$ poemas que vienen en castellano; quechuas con sus transcripciones al español y las del folklore, recopilaciones de canciones que se inscriben en quechua, se traducen a la lengua de la ciudad. Casi una década después, aparece Taki parwa (1955) de Kilko Warak'a, tiene el mérito de ser el primer libro orgánico de poesía quechua en los tiempos modernos. Al año siguiente, 1956, Kusi Paukar publica Jarawikuna enteramente en quechua chanka en la Revista Cultura de Bolivia (Huamán Águila 2017).

Como se aprecia en el Cuadro 1 ponemos el énfasis al proceso. Esto es entender las dinámicas sociales y su histórica en su dimensión contradictoria. Pacha waray, Primeras muestras: 1904-1947, toma como referencia la publicación de Aurora / Pacha huarai (1904), aparecen de manera esporádica en revistas. De este periodo destacamos D.S.V., Moisés Cavero Caso, Inocencio Mamani, José Salvador Cavero, Teodoro Meneses, Porfirio Meneses Lazón, Reynaldo Martínez Parra y Juan de la Cruz Salas. Kunankaq qallarichiqkuna, Fundadores de la tradición (1948-1962) se caracteriza porque la poesía alcanza autonomía. La poesía que producen alcanzan autonomía poética, dialogan con otras tradiciones y se preserva el pensar andino; usan o no el seudónimo, escriben en quechua, prefieren o no traducir. Sintetizan la poesía quechua de estos tres poetas fundacionales: Kilko Warak’a, Kusi Paukar y José María Arguedas.

5 Audaz Castillo corresponde al seudónimo del «Poeta ermitaño i apasionado romántico del lago de Langui-Layu, don José Cirilo del Castillo i Gutiérrez. [1895]» que publicó la revista Wikuña (1929); Kilko Warakca, corresponde a Andrés Alencastre; Tupac-Amaro (Canas-Surimane, 1915-?) es seudónimo de "J. de la Cruz i S, autodidacta, tipógrafo i chofer.», y, Jacinto Yana-Aucca es identificado como «poetas netamente INDIO don Jacinto Ccallo y Sumi [1923?], oriundo de Yanaoca.» (cf. Tupak Amaro 1947: s/n).
Musuqyaywan upallasqa, lo definido como momento de «renovación» y «silencio» abarca 19631989, comprende el periodo en que los idiomas vernáculos y la escritura quechua congenian con la euforia nacionalista hasta el momento en que el interdicto de la runasimi por efecto de la guerra interna. Incluyo dos momentos: el primero «Euforia e identidades quechuas» $\mathrm{y}$ "Los ochenta», segundo momento, tiene creadores que habrán de silenciarse en medio del conflicto armado interno. Destacan William Hurtado de Mendoza, Issaac Huamán Manrique, Eduardo Ninamango Mallqui y Dida Aguirre García Harawikunataq kutimanchisqa (1990-2000) marcado por los retornos y presencias, corresponde a la última década del siglo xx. Divido a esta sección en tres grupos aglutinadores: El primero, "Convergencias", en que aparecen poetas que no configuran un núcleo de referencias y pero publican durante el periodo. El segundo, "Los qantus», momento en que el quechua reaparece como escritura y retoma su difusión, tiene dos nudos de desenlace. El tercero, lo identifico como: «Quechuas trans-andinos» que a fines del siglo Xx viven un proceso paradojal, que coincide con las políticas del fin de la historia, en ese mismo espacio de las simultaneidades aparece un núcleo que sabe utilizar adecuadamente las nuevas redes de comunicación, especialmente las virtuales, y tiene que ver con poetas que asumen una perspectiva abierta que inciden en nuevas posibilidades poéticas. Entre ellos advertimos el astro poético de Baltazar Azpur Palomino, Gloria Cáceres Vargas, Víctor Tenorio García, José Antonio Sulca Effio; Odi Gonzales, Wilbert Pacheco Alvarez y Fredy A. Roncalla.

Las secciones Napumantawan Ankashmanta y Yachaqkuna (Escoleros) escapan a la propuesta, expresan la diáspora y la diversidad poética quechua. La primera al quechua amazónico, en este caso las poéticas orales que fueron llevadas a las letras del quechua del Napo y la segunda presenta al quechua ancashino en su poesía, que escasamente circula en los espacios literarios (César Vargas Arce, Domingo Sigüeñas Vivar, Didí Cadillo Rosas y Macedonio Villafán Broncano. Y Yachaqkuna (Escoleros) propone una mirada a las formas de divulgación del quechua, reúno la poesía que se divulga principalmente en y como textos escolares en las escuelas.

Iskay waranqakuna (Los, las, del 2000 -) corresponden a la consolidación de la poesía que- 
Cundro 2. Publicaciones de Iskay waranqap, La primera década

\begin{tabular}{|c|c|c|c|c|c|c|c|c|c|c|c|}
\hline Autor & Lugar de nacimiento & 2004 & 2005 & 2006 & 2009 & 2010 & 2014 & 2016 & 2017 & 2018 & 2019 \\
\hline $\begin{array}{ll}\text { - } & \text { Edgar } \\
\text { HunanCachoque } \\
\text { Nieto }\end{array}$ & Cusco & & 1 & 1 & & & & & & & \\
\hline $\begin{array}{ll}\text { - } & \text { Gladis Naty } \\
& \text { Valencia Rosell } \\
\end{array}$ & Cotabambas (Apurímac) & & & & & & & & & & \\
\hline - Jesús del ANde & Cusco (?) & & & & & & 1 & & & & \\
\hline $\begin{array}{l}\text { - Lenon Tomás } \\
\text { Tutaya de la Cruz }\end{array}$ & $\begin{array}{l}\text { Tarwiqucc, Acocro, } \\
\text { (Ayacucho, 1997) }\end{array}$ & & & & 1 & & & & & & \\
\hline $\begin{array}{l}\text { - Washington } \\
\text { Córdova Huamán }\end{array}$ & $\begin{array}{l}\text { Circa, Abancay (Apurímac, } \\
\text { 1962) }\end{array}$ & & & & & 1 & & & & 1 & 1 \\
\hline $\begin{array}{ll}\text { - Leonidas CaSas } \\
\text { Ballón } \\
\end{array}$ & Mollepata (Apurímac, 1949) & & & & & & & 1 & & & \\
\hline $\begin{array}{l}\text { - Ch'aska Eugenia } \\
\text { Anka Ninawaman }\end{array}$ & Nacida en Cusco (1973) & 1 & & & & 1 & 1 & & & & \\
\hline $\begin{array}{l}\text { - Ugo Facundo } \\
\text { Carrillo Cavero }\end{array}$ & $\begin{array}{l}\text { Nació en la Comunidad de } \\
\text { Uripa (Abancay, 1956) }\end{array}$ & & & & 1 & 1 & & & & & \\
\hline $\begin{array}{l}\text { - Carlos Huamán } \\
\text { López }\end{array}$ & Ayacucho (1958) & & & & 1 & & & & & & \\
\hline
\end{tabular}

chua contemporánea. Hay una diáspora entre los que publican tardíamente y los jóvenes que publican, entre los que tienen la pericia y experticia de la vida y la lectura, y aquellos que tienen un pedazo de existencia y universos de sensibilidad que desean volcarlo al poema. "La primera década» incluye a poetas que han publicado en diversas revistas, sea en Cusco, Huamanga o Lima (Ch'aska Eugenia Anka Ninawaman, Hugo Carrillo y Carlos Huamán López). Y «Los penúltimos» forman parte de diversos circuitos literarios (Nora Alarcón, Niel Palomino, Percy Borda, Yuly Tacas) donde su poesía circula, ellos mismos son difusores a través de espacios poéticos (Rubén Yucra, Edwin Ramos), concursos (Wendy Bellido) o publicaciones como revista en quechua (Atuqpa chupan, Kallpa), se corresponde con esta cartografía las poéticas quechuas de Cleofé Prado Huamán, Jesús Cárdenas Huayllaro y Olivia Reginaldo.

\section{Conclusiones}

Tenemos por cierto que el aprendizaje poético parte de una práctica de escritura y publicaciones en castellano. La experiencia literaria viene subyugada por la ciudad letrada, antes de hacerlo en quechua la mayoría de los poetas primero publican en español.
De esta suerte consideramos que el protocolo anota básicamente el momento en que se hace evidente las producciones poéticas quechuas en circuitos locales o nacionales. Nuestra poesía tiene sus referentes en la cultura oral quechua que precede a la escritura alfabética.

Como proceso encontramos que los primeros poemas del siglo xx se publican en Auroral Pacha Huarai, edición eventual "(Escrito en dialecto tarmeño)»; le sigue la discontinua y episódica presencia de poemas en lenguas indígenas en publicaciones periódicas. Lo que llamaremos partida de nacimiento de la poesía quechua escrita contemporánea, su tradición moderna, del siglo xx tiene lugar en el tramo 19471956, cuyos textos fundacionales corresponden a las publicaciones de Canas i sus relámpagos (1947), Taki parwa (1955) y Harawikuna (1956). La poesía quechua acusa una doble dinámica como parte de la conciencia poética. Expresarse solo en la lengua (ñuqaku) o hacerlo en esta y al mismo tiempo tender un puente para el hablante castellano (ñuqanchik).

La producción fundacional se concentra en tres autores, Andrés Alencastre, César Guardia Mayorga y José María Arguedas en el momento en que el quechua era despreciado por ser cosa de indios. Este grupo de poeta le otorga modernamente autonomía a la poesía quechua escrita. Luego vendrá un proceso al- 
rededor de la euforia y vindicación del campesino andino, con la oficialización de quechua. En los años 80 se producirá un silencio. No circulan los poemas quechua ni aparece en en los escenarios públicos, exactamente porque una vez más esta lengua y su gente son catalogados indios, se la discrimina, es asunto de amenaza, se la convierte en asunto de guerra. Luego de este ingrato episodio que tiene lugar en el país aparece la vindicación de la poesía quechua a partir de los núcleos de escritores en el caso de Ayacucho y en caso de Cusco a partir de las instituciones. Aparecen voces que hasta entonces circulaban en espacio local sin una mayor dinámica entre los circuitos culturales, que da lugar a lo que sucederá en el siglo xx, el fortalecimiento del quechua. El siglo XxI se caracterizará precisamente por eso por una mayor capacidad para la circulación de las poéticas quechua, incluida publicaciones académicas en la lengua como Atupaq chupan o la utilización de las plataformas virtuales. La literatura se establece en un circuito público, no privado ni íntimo; la misma que le permite construirse el hecho poético.

Finalmente, se trata de lo que formalmente ha quedado, una poesía que asume la aventura poética como desafío. No se agota en la ruta insólita cuando se vuelve fronteriza. Escribir como quechua en castellano o transitar del quechua al español y de este al inglés en un conglomerado sémico andino o hacerlo en la misma lengua (del quichua al quechua cusqueńo). Sigue siendo una palabra articuladora y que se renueva constantemente, que aparenta el rumor local y tiene el empuje de una poesía que va más allá de las fronteras, una poesía que cuya lectura nos deje la extrańeza poética. 


\section{Bibliografía}

Alejandro Romualdo (1984). Poesia aborigen y tradición popular. Lima: Ediciones Edubanco, (Poesía Peruana, Antología General, t. I).

Alencastre, Andrés (Kilko Warak'a). «Puma». En Waman Puma, ańo I, vol. I, N. ${ }^{\circ}$ 1, Cusco, octubre de 1941; p. 9.

Álvarez, Rolando (2016). Adentro de la noche. Una lectura de las novelas El tungsteno de César Vallejo y El Sexto de José María Arguedas desde la estética, la historia y la crítica. Guanajuato (México): Catedra José Revuelta Universidad de Guanajuato.

Ángeles Caballero, César (1978). «La poesía ayacuchana» en Literatura Peruana. Ayacucho. Lima, t. III; pp- 73-108.

Arguedas, José María (2012). Obra antropológica. Lima: Editorial Horizonte - Comisión Centenario del Natalicio de José María Arguedas; 7 t.

Bendezú Aybar, Edmundo (1980). Literatura Quechua. Edición, prólogo y cronología de Edmundo Bendezú. Caracas: Biblioteca Ayacucho; vol. 78.

Carrillo, Francisco (1968). Poesía y prosa quechua. 2a ed. Prólogo de José María Arguedas. Selección de Francisco Carrillo. Lima: Ediciones de la Biblioteca Universitaria.

Cavero, Moisés C. (1938). "iHuaccha huahuaiquim cani!.../ ¡Huyarihuai!...», en Huamanga, n. 12: p. 16-18.

Chillcce Canales, Edwin (2017). La poética chanka en tres poemarios. Tesis de magíster. Lima: Universidad Nacional Mayor de San Marcos.

Cornejo Polar, Antonio (1983). «La literatura peruana: totalidad contradictoria», Revista de Crítica Literaria Latinoamericana IX, 18, Lima: 37-50.

Espezúa Salmón, Dorian (2017). Las consciencias lingüisticas en la literatura peruana. Lima: CELACP, Lluvia Editores.

Espino RelucÉ, Gonzalo (2015). «Ñuqa-ñuqanchis: Polos de desarrollo poético, resistencia colectiva y ego andino en la poesía quechua contemporánea (1982-2014)», en Escritura y Pensamiento, vol. 18, n. 37: 53-78.

Espino Relucé, Gonzalo (2007). Etnopoética quechua: textos y tradición oral quechua. Tesis doctoral. Lima: Universidad Nacional Mayor de San Marcos.

Garcilaso de la VeGa, Inca (1985 [1609]). «De los amautas y la poesía en los tiempos de los incas» en Los comentarios reales de los Inca. Lima: Banco de Créditos del Perú; pp. 87-90.
Guillén, Paul (2016). Aguas móvles. Antología de la poesía peruana 1978-2006, Lima: Perro de ambiente editor.

Huamán Águila, Óscar (2007). La pervivencia de la identidad cultural como memoria del tiempo moderno en Sonqup Jarawiinin, Umapa Jamutaynin, Runap Kutipakuynin de Kusi Paukar. Tesis de licenciatura. Lima: Universidad Nacional Mayor de San Marcos. http://cybertesis.unmsm.edu.pe/bitstream/ cybertesis/6738/2/Huaman_ao.pdf

Huamán López, Carlos (2015). Urpischallay. Transfiguraciones poéticas, memoria y cultura popular andina en el wayno. México/Lima: Universidad Autónoma de México, Ediciones Altazor.

Huamán Manrique, Isaac (2018). La poesía quechua escrita en Huancavelica. Reconstrucción de una identidad. Con un estudio sobre la poesía quechua escrita en el Perú. Lima: La Escuelita de América.

Huamán Manrique, Isaac (1996). La poesía quechua escrita actual (1990-1995). Tesis de Licenciatura. Lima: Universidad Nacional Mayor de San Marcos.

INDio ENILDA (1946). "iSayhulla rumil», en Huamanga, n. ${ }^{\circ}$ 63: p. 29.

Kilko Waraka (1942). «Ayakuchokc, napaycuykin» / "Ayacucho yo te saludo», Waman Puma, año II, vol. II, N.o 3- 4. Cusco, diciembre 1941 - enero; p.33; p. 68.

Kusi Paukar [César Guardia Mayorga] (1956). "Modalidades expresivas del kechwa» y "Jarawikuna» en Revista de Cultura, $\mathrm{n}^{\circ}$ 2. Cochabamba, diciembre; pp. 321-327, 327-332.

LienHARD, Martín (1998). «Pachakutiy taki: canto y poesía quechua de la transformación del mundo» en Oralidad, vol. 9 (La Habana): 30-41. http://unesdoc.unesco.org/images/0011/001129/112971so. pdf\#nameddest $=112953$

Mamani Macedo, Mauro; Mamani Mamani, Alan Ever (2017). Harawikuna Jarawinaka. Quechua-Aymara. Lima: ESANDINO-Lluvia Editores.

Ninamango Mallqui, Eduardo (1982). Katatay y la poética quechua de José María Arguedas. Tesis de Licenciatura. Lima: Universidad Nacional Mayor de San Marcos.

Noriega Bernuy, Julio E. (2012). Camina los Apus. Escritura andina en migración. Lima: Pakarina Ediciones, Knox College.

Noriega Bernuy, Julio E. (2016). Poesía quechua escrita en el Perú. Antología. Lima: CEP Centro de Estudios Publicaciones, 1993. 2a ed. Cusco: Ministerio de Cultura, Dirección Desconcentrada de Cultura de Cusco. 
Rodríguez Monarca, Claudia (2017). «Dinámicas de contacto entre la producción poética andina (kichwa y aymara) y el canon literario», Letras 88 (127): 31-54.

Salazar Bondy, Sebastián (1978). Poesía quechua. Traducción y notas de Sebastián Salazar Bondy. Buenos Aires: Ed. Arca/ Ed. Galerna.
Tupak-Amaro (1947). Canas i sus relámpagos. Antología poética. Cusco: Tip. El Inca.

Zevallos Aguilar, Ulises Juan (2009). Las provincias contraatacan. Regionalismo y anticentralismo en la literatura del siglo xx. Lima: Ediciones del Vicerrectorado Académico de la Universidad Nacional Mayor de San Marcos. 